\title{
Graphical User Interface Design for Collaborative Virtual Environments
}

\author{
M. K. Othman \\ Faculty of Cognitive Sciences and Human Development (FCSHD) \\ Universiti Malaysia Sarawak (UNIMAS) \\ Sarawak, Malaysia
}

\begin{abstract}
The project aims to study, evaluate, analysis a Collaborative Virtual Environments (CVE's) software also known as Networked Virtual Environments (NVE's), and suggest how to improve the existing user interface by designing an alternative graphical user interface (GUI) for CVE's software. To reduce cost, most current and operative CVE's use the Internet and standard PC to create a visual Virtual Environment (VE), which can be shared by a large number of users. This project discusses $C V E$ 's and the importance of using a good GUI for a CVE system.
\end{abstract}

\section{INTRODUCTION}

The aim of this research is to study and evaluate Collaborative Virtual Environment (CVE) also known as Networked Virtual Environments (NVE) software and suggest a better or suitable Graphical User Interfaces. This will include a software analysis and evaluation, updating the software if and when necessary or is possible. Outcome of this project is important for improvement of better CVE software in the next phase. It will discuss what has been gathered from the research and the analysis of the CVE user interfaces. It also discusses and addresses some of the issues from the research.

In this project, user interface not only refers to the graphical interface (Graphical User Interface ${ }^{l}(G U I)$ ) between the user and the system, but includes other interfaces for communication such as aura communication, voice or video interface communication, facial expression and also standard tools used for communication (e.g. keyboard, mouse, etc).

The CVE software in this report refers to the software designs by Oliver Wennel at School of Computing Sciences, University of East Anglia United Kingdom.

\footnotetext{
${ }^{1}$ A graphical user interface (or GUI, pronounced "gooey") is a method of interacting with a computer through a metaphor of direct manipulation of graphical images and widgets in addition to text. The GUI was invented by researchers at the Stanford Research Institute (including Doug Engelbart) for use in its On-Line System [1].

1-4224-0220-4/06/\$C 2006 IEEE.
}

\section{BACKGROUND STUDY}

A Collaborative Virtual Environment (CVE) can be defined as a tool that supports collaboration between multiple users over a computer network. According to Churchill et al. [2] a CVE is a computerbased, distributed, virtual space or set of places. In such places, people can meet and interact with each other, with agents or with virtual objects. A CVE might vary in its representational richness from 3D graphical spaces, 2.5D and 2D environments, to testbased environments. Access to CVE's is by no means limited to desktop devices, but might well include mobile or wearable devices, public kiosks, etc.

This study focuses on a $3 \mathrm{D}$ virtual world of CVE systems. A CVE can provide a more naturally shared system, by supporting interactive human collaboration and integrating different media in real time in a single $3 \mathrm{D}$ surrounding [3]. Nowadays, a CVE is not only visible to research laboratories with expensive equipment, but it could also be accessed by the public via the Internet.

Examples of CVE systems available on the market are DIVE [4], MASSIVE [5], NPSNET [6], SPLINE [7], etc. Each system has its own strengths and weaknesses. With the enhancement of current technologies, it could not be denied that a CVE could support collaborative work. However to make it work in practical conditions, customized design and development are necessary. Some of these issues will be discussed in detail in the next part of this report, in particular the user interface design in a CVE system.

Human Computer Interaction (HCI) is a study of how humans interact with computers, and how to design computer systems that are easy, quick and productive for humans to use [8]. Research in HCI has changed the way user interfaces are currently designed. However, research in this area has not only focused on the design of user interfaces but is also concerned with human factors as a consequence of using the system.

HCI could be defined as the study, evaluating, planning, and design of what happens between a user and a computer when they are working together [9]. In order to enable an interaction between human and computer, the interaction must consist of at least three 